\title{
Body Surface Area Is Not a Reliable Predictor of Tracheal Tube Size in Children
}

\author{
Filiz Uzumcugil · Emre Can Celebioglü ${ }^{2, \star} \cdot$ Demet Basak Ozkaragoz ${ }^{1} \cdot$ Aysun Ankay Yilbas $^{1} \cdot$ Basak Akca $^{1}$ \\ Nazgol Lotfinagsh ${ }^{1} \cdot$ Bilge Celebioglu ${ }^{1}$ \\ ${ }^{1}$ Department of Anesthesiology and Reanimation, Hacettepe University School of Medicine, Ankara; \\ ${ }^{2}$ Department of Radiology, Karabük University, Karabük, Turkey
}

Objectives. The age-based Cole formula has been employed for the estimation of endotracheal tube (ETT) size due to its ease of use, but may not appropriately consider growth rates among children. Child growth is assessed by calculating the body surface area (BSA). The association between the outer diameter of an appropriate uncuffed-endotrachealtube (ETT-OD) and the BSA values of patients at 24-96 months of age was our primary outcome.

Methods. Cole formula, BSA, age, height, weight and ultrasound measurement of subglottic-transverse-diameter were evaluated for correlations with correct uncuffed ETT-OD. The Cole formula, BSA, and ultrasound measurements were analyzed for estimation rates in all patients and age subgroups. The maximum allowed error for the estimation of ETT-OD was $\leq 0.3 \mathrm{~mm}$. Patients' tracheas were intubated with tubes chosen by Cole formula and correct ETT-OD values were determined using leak test. ETT exchange rates were recorded.

Results. One-hundred twenty-seven patients were analyzed for the determination of estimation rates. Thirteen patients aged $\geq 72$ months were intubated with cuffed ETT-OD of $8.4 \mathrm{~mm}$ and were accepted to need uncuffed ETT-OD $>8.4$ $\mathrm{mm}$ in order to be included in estimation rates, but excluded from correlations for size analysis. One-hundred fourteen patients were analyzed for correlations between correct ETT-OD (determined by the leak test) and outcome parameters. Cole formula, ultrasonography, and BSA had similar correct estimation rates. All three parameters had higher underestimation rates as age increased.

Conclusion. The Cole formula, BSA, and ultrasonography had similar estimation rates in patients aged $\geq 24$ to $\leq 96$ months. BSA had a correct estimation rate of $40.2 \%$ and may not be reliable in clinical practice to predict uncuffedETT-size.

Keywords. Body Surface Area; Endotracheal Intubation; Pediatrics; Ultrasonography

\section{INTRODUCTION}

In pediatric patients, the estimation of endotracheal tube (ETT) size is still controversial. Anatomical, structural, and develop-

\footnotetext{
- Received February 6, 2018

Revised March 20, 2018

Accepted June 27, 2018

- Corresponding author: Filiz Uzumcugil

Department of Anesthesiology and Reanimation, Hacettepe University

School of Medicine, Sihhıye, Ankara 06230,Turkey

Tel: +90-3123051265, Fax: +90-3123051260

E-mail: filizuzumcugil@hotmail.com

*Emre Can Celebioglu is now working at Karabük University. The study was conducted at Hacettepe University.
}

mental investigations have resulted in age-, height- and weightbased associations with developmental and structural alterations in the pediatric airway [1-3]. In terms of airway management, ETT size estimations still require methods that allow rapid and accurate decisions.

The most rapid method for the determination of ETT size in pediatric patients depends on age. Age-based recommendations are used in infants and children. More specifically, the Cole formula [inner diameter of ETT $=($ age $/ 4)+4]$ is recommended for children $>2$ years ( $>24$ months) of age [4]. The Cole formula has been reported to have a correct prediction rate of $47 \%-$ 77\% [5-7]. Height-based estimations such as the Broselow tape and formulas including height, weight, and age have been uti-

Copyright $@ 2018$ by Korean Society of Otorhinolaryngology-Head and Neck Surgery

This is an open-access article distributed under the terms of the Creative Commons Attribution Non-Commercial License (http://creativecommons.org/licenses/by-nc/4.0)

which permits unrestricted non-commercial use, distribution, and reproduction in any medium, provided the original work is properly cited. 
lized $[6,8,9]$. However, such formulas have been suggested to be of limited use due to a lack of estimation for individual variations in the growth of internal organs [6].

The growth of children is assessed by considering both weight and height and their correlated increase with age. The percentile charts for weight-for-age and height-for-age are used to monitor growth. Children who are at $<5 \%$ for weight-for-age and heightfor-age may have smaller tracheal dimensions with respect to their age. Therefore, the Cole formula may be more misleading in these children. Despite the limitations of their study, Daugherty et al. [10] investigated children with pathologically short stature (PSS; $<5 \%$ height-for-age) for the estimation of correct ETT size, and reported that height-based predictions were at least as accurate as age-based predictions in both PSS and normal children [10]. A more precise method including both weight and height for the assessment of the growth of a child involves calculating the body surface area (BSA) [4]. In our study, BSA was investigated for its advantage of including two major parameters (weight and height) that are used to monitor growth in children. In a recent study, Neunhoeffer et al. [11] created new diagrams based on BSA to predict the correct tube length and reported that the rate of correction for the placement of an ETT decreased after employing these diagrams. In investigations addressing the structure of the pediatric airway, the transverse diameter of the subglottic region was reported to be the narrowest of all regions and was found to be positively correlated with the height and the cross-sectional areas of both the glottis and the cricoid, which were found to be positively correlated with age, height, and weight $[2,3]$. In addition, the cross-sectional area of these two regions was reported to closely approximate each other with increasing growth [3]. Considering these data and the non-linear increases in height, weight and surface area in children with respect to age, we thought that the tracheal dimensions may be affected by individualized growth and the speed of that growth. Therefore, to include the main parameters that are used to monitor the growth of a child, we aimed to determine the impact of BSA on the correct outer diameter of an appropriate uncuffed-endotracheal-tube (ETT-OD) compared to the age-based Cole formula.

In recent years, ultrasound has proven to have a higher rate of prediction of the correct ETT size in pediatric patients than any other frequently used methods [5-7]. Our first objective was to investigate the association between the outer diameter of the

\section{H I G H L I G H T S}

- The Cole formula, body surface area and ultrasonography had similar estimation rates in patients aged $\geq 24$ to $\leq 96$ months.

- Body surface area had a correct estimation rate of $40.2 \%$ and may not be reliable in clinical practice to predict uncuffed-endotracheal tube size. correct ETT and BSA in a group of pediatric patients between 24 and 96 months of age. Our second objective was to determine the associations between age, height and weight as predictors of the outer diameter of the correct ETT and to determine whether percentiles of weight- or height-for-age have an impact on tube exchanges. Our third objective was to compare the associations between the Cole formula, BSA and the ultrasound measurement of the transverse diameter of the subglottic area with the correct ETT size in subgroups by age in patients aged 24-96 months.

\section{MATERIALS AND METHODS}

After obtaining approval from the Institutional Ethical Board Committee (No. 14/124-12; 30.04.2014) and written informed parental consent, 24 to 96-month-old American Society of Anesthesiology (ASA) physical status grade I-II patients, undergoing elective surgical procedures (for which endotracheal intubation was indicated) between March 1, 2016 and August 31, 2016 (6-month period) were examined for study purposes. Patients with known or suspected laryngeal or tracheal pathologies or syndromes characterized by airway anomalies or difficult airways were not included. Data on age (years and months), body weight and body height (measured the day before surgery) were obtained from records and BSA was calculated using the Mosteller formula $\left\{\right.$ BSA $\left(\mathrm{m}^{2}\right)=\sqrt{ }\left[\right.$ weight $(\mathrm{kg}) \times$ height $\left.\left.(\mathrm{cm}) 3,600^{-1}\right]\right\}$ [12]. Percentiles pertaining to weight- and height-for-age were determined using growth charts (Centers for Disease Control and Prevention [CDC 24/7]) [13].

All patients received standardized anaesthetic management. All patients received oral/nasal administration of $0.5 \mathrm{mg} / \mathrm{kg}$ midazolam. Electrocardiography, noninvasive blood pressure, and peripheral oxygen saturation were monitored. Anaesthetic induction was provided by sevoflurane. After obtaining intravenous access, $1 \mathrm{mg} / \mathrm{kg}$ of propofol and $1 \mu \mathrm{g} / \mathrm{kg}$ of fentanyl were administered and $0.6 \mathrm{mg} / \mathrm{kg}$ rocuronium was used for muscle relaxation.

Ultrasound measurements (40 mm linear probe, $12-7 \mathrm{MHz}$; Titan, Sonosite, Bothell,WA, USA) were performed before intubation and after obtaining paralysis. The probe was first used to locate the hyoid cartilage, which was then moved caudally to locate the true vocal cords, and the subglottic transverse diameter was obtained immediately below the vocal cords. The measurements were performed by briefly interrupting positive pressure ventilation (without positive end-expiratory pressure) to minimize alterations in airway diameter for less than 30 seconds. The measurements were performed by the same anaesthesiologist (FU) after a supervised learning period. Endotracheal intubations were performed using direct laryngoscopy and an uncuffed ETT, and the tube sizes were determined using the Cole formula: inner diameter $(\mathrm{ID})=($ age $[$ years] $/ 4)+4$. The ETT sizes were as 
follows: (ID/OD)=4.0/6.1, 4.5/6.6, 5.0/7.1, 5.5/7.7, and 6.0/8.4 $\mathrm{mm}$ (Bicakcilar, Istanbul, Turkey). If the tube size obtained from the Cole formula was between two sizes, then the smaller tube was selected. During the first attempt, if resistance was encountered, the tube was exchanged for a tube that was $0.5 \mathrm{~mm}$ smaller. The depth of the tracheal tube was confirmed by auscultation, and ventilation was examined via tracheal leak. Respiratory pressures were slowly increased (provided that the pressure did not exceed $25 \mathrm{cmH}_{2} \mathrm{O}$ ) to identify an audible leak from the patient's mouth. If there was an audible leak with an airway pressure $<10 \mathrm{cmH}_{2} \mathrm{O}$, the tube was exchanged for one that was $0.5 \mathrm{~mm}$ larger. If there was no leak with a pressure $>20 \mathrm{cmH}_{2} \mathrm{O}$, the tube was exchanged for one that was $0.5 \mathrm{~mm}$ smaller. An audible leak between 10 and $20 \mathrm{cmH}_{2} \mathrm{O}$ was only acceptable if ventilation was adequate. Otherwise, if the leak was excessive and did not allow adequate ventilation with plateau-type end-tidal capnography, the tube was exchanged for one that was $0.5 \mathrm{~mm}$ larger. In patients who had an excessive air leak with an uncuffed tube with an ID of $6.0 \mathrm{~mm}$ (OD, $8.4 \mathrm{~mm}$ ), the tube was exchanged for a cuffed tube with an ID of $6.0 \mathrm{~mm}$ instead of exchanging with an uncuffed tube with an ID of $6.5 \mathrm{~mm}$. Because uncuffed ETTs are not preferred in children over 8 years of age, for which the Cole formula concludes on an uncuffed ETT with an ID of $6.0 \mathrm{~mm}$. Therefore, patients who needed an uncuffed ETT with an OD larger than $8.4 \mathrm{~mm}$ (ID, $6.0 \mathrm{~mm}$ ) were intubated with a cuffed ETT with an OD of $8.4 \mathrm{~mm}$. These patients were excluded from the correlations with size and were included in the analyses of tube-exchange rates and size estimation rates. The correct uncuffed ETT sizes for these patients were accepted as OD $>8.4 \mathrm{~mm}$ for estimation rates. Since the inflated cuff would not reflect the exact size, these patients were not included in the analysis for correlations with size. The correct tube outer and inner diameters (correct ETT-OD and ID) and the tube exchanges were recorded. If an ETT was exchanged more than twice, $1 \mathrm{mg} / \mathrm{kg}$ of intravenous methylprednisolone was administered. Ultrasound measurements and examinations for tracheal leak were performed with the patient in the supine position with the head and neck in neutral position.

The Cole formula was compared to the correct ETT-ID because the formula estimates inner diameter. Similarly, because ultrasound measures the tracheal diameter to fit the outer diameter of the ETT, comparisons were performed using the correct ETT-OD. All correlations were performed using the correct ETTOD. Considering that adequate ventilation is provided by a sufficient seal that is established by the tracheal mucosa and the ETT, a sufficient seal depends on the outer diameter of the ETT. Moreover, outer diameter matched inner diameter changes depending on the manufacturer. Therefore, the outer diameter was used for the correlations.

The subgroups of age were determined in terms of months: ages $\geq 24$ to $<48, \geq 48$ to $<72$ and $\geq 72(\geq 72$ to $\leq 96)$ months. The groups were determined according to the tube sizes esti- mated by Cole formula; 24 months of age ( 2 years), 48 months of age (4 years), 72 months of age ( 6 years) and 96 months of age (8 years) were to be intubated with ETTs of internal diameters of $4.5 \mathrm{~mm}, 5 \mathrm{~mm}, 5.5 \mathrm{~mm}$, and $6 \mathrm{~mm}$, respectively. Hence, there were only two size possibilities for each subgroup. If the age was in between according the tube size, we preferred the smaller tube as mentioned before.

The primary aim of this study was to determine the effect of BSA on correct ETT-OD. A total sample size of 102 was required to detect the correlation between BSA and ETT-OD after adjusting for age with a power of $95 \%$ at a $5 \%$ significance level. The sample size estimation was performed using G*Power ver. 3.0.10 (Universitat Kiel, Kiel, Germany). After calculations for drop-out, a sample size of 127 was defined.

\section{Statistical analyses}

Descriptive statistics are shown as the number of cases and percentages for categorical data. The mean \pm standard deviation or the median percentiles (25th-75th) were used for continuous variables. Whether the mean differences between clinical measurements were statistically significant was evaluated with a paired sampled $t$-test. Degrees of association between continuous variables were tested using a Pearson moment correlation analysis. Bland-Altman plots were used to assess the magnitude of the disagreement between the correct ETT-OD and ultrasound measurements and between the correct ETT-ID and the Cole formula. The means of agreement differences (i.e., bias) and the lower and upper limits set at a 0.95 confidence interval were also calculated. The maximum allowable deviation of the measurements from the correct ETT-OD or ID was $\leq 0.3 \mathrm{~mm}$ [5]. Univariate linear regression analyses were applied to determine the effect of each independent variable on the correct ETTOD. Coefficients of regression, $95 \%$ confidence intervals and $t$ statistics for each independent variable were also calculated. The differences in estimation rates (i.e., under-, correct- and over-estimates) between indicators (i.e., Cole formula vs. BSA and Cole formula vs. ultrasonography were analyzed by the McNemar test. The data analysis was performed by using MedCalc ver. 11.1.1.0 (Broekstraat 52, B-9030; MedCalc software, Ostend, Belgium). A $P$-value less than 0.05 was considered statistically significant.

\section{RESULTS}

In this study, we evaluated 127 patients who were 24-96-months old, with ASA grades of I-II, who were scheduled for surgical procedures in which endotracheal intubation was indicated. A total of 13 patients required an ETT with an ID larger than $6.0 \mathrm{~mm}$ and were intubated with a cuffed ETT with an ID of $6.0 \mathrm{~mm}$. These patients were in the $\geq 72$ to $\leq 96$ months age group and were excluded from the size analysis but have been presented 
as percentages in size estimation rates. The data of 114 patients were included in the size analysis (Fig. 1). Demographic data are shown in Table 1. The correct uncuffed ETT-ODs used are shown in Table 2. The most frequently used uncuffed ETT-OD for each subgroup of age is highlighted (Table 2).

In Table 3, the effects of weight (per $1 \mathrm{~kg}$ ), height (per $10 \mathrm{~cm}$ ) and BSA (per $0.1 \mathrm{~m}^{2}$ ) in each subgroup of age are given. All three parameters were effective for tube size estimation in all

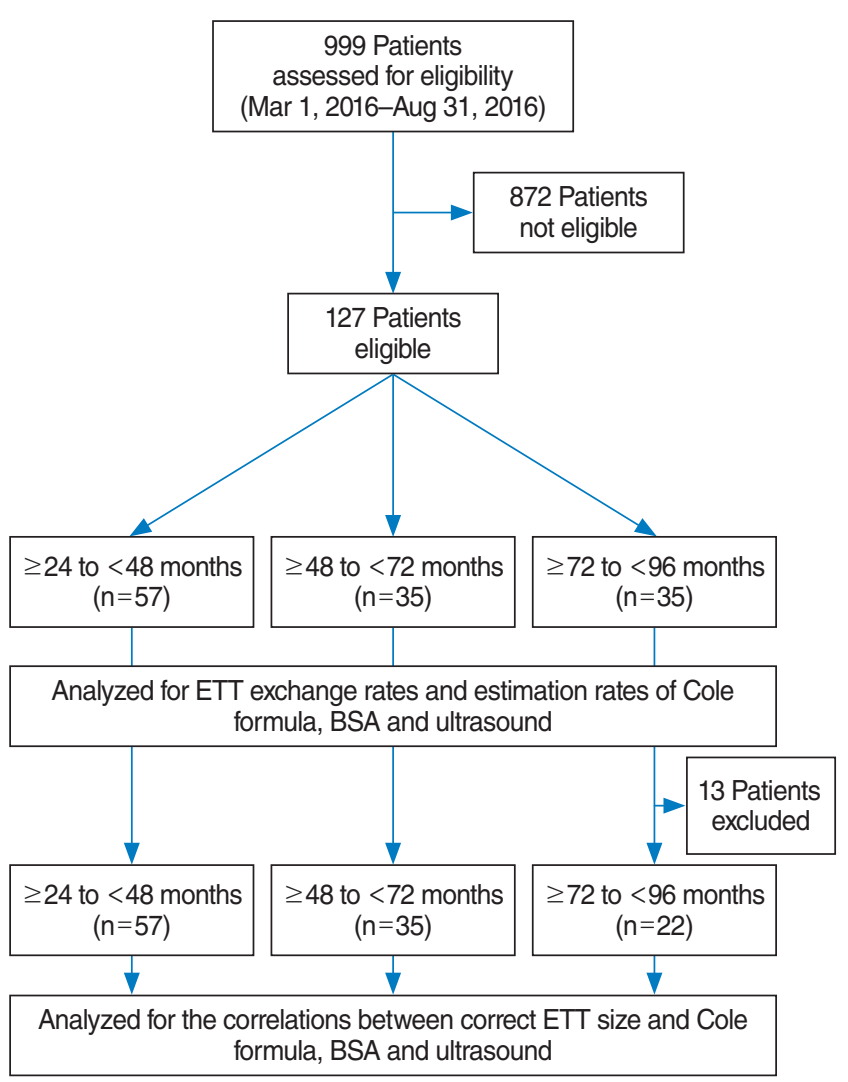

Fig. 1. Flowchart describing patients scheduled for surgery, and assessed and analyzed for the study within a 6-month period. ETT, endotracheal tube; BSA, body surface area.

Table 1. Demographical data

\begin{tabular}{lc}
\hline Variable & Value $(\mathrm{n}=114)$ \\
\hline Age $(\mathrm{mo})$, mean \pm SD $($ range $)$ & $50.4 \pm 19.1(24-96)$ \\
Sex & $85(74.6)$ \\
$\quad$ Male & $29(25.4)$ \\
Female & $16.4 \pm 3.9$ \\
Weight $(\mathrm{kg})$ & $104.5 \pm 11.6$ \\
Height $(\mathrm{cm})$ & $0.69 \pm 0.12$ \\
BSA $\left(\mathrm{m}^{2}\right)$ & $0.50(0.10-0.75)$ \\
Weight percentile for age & $0.75(0.25-0.90)$ \\
\hline Height percentile for age &
\end{tabular}

Values are presented as number (\%), mean $\pm \mathrm{SD}$, or median (range) unless otherwise indicated.

SD, standard deviation; BSA, body surface area. subgroups of age except for weight in children at the age of $\geq 48$ to $<72$ months $(P=0.119)$ (Table 3).

In all patients (age $\geq 24$ to $\leq 96$ months) and in all subgroups of age the correct estimation rates of the Cole formula, BSA and ultrasound were similar (Table 4 ). In $\geq 24$ to $<48$ months age patients, the under-estimation rates of the Cole formula were higher $(P=0.021)$ compared to BSA, but the under-estimation rates were similar with ultrasound $(P=0.167)$. The over-estimation rates of the Cole formula were lower than both BSA $(P=0.021)$ and ultrasound $(P=0.002)$. In $\geq 48$ to $<72$ months age patients, despite statistical insignificance, the under-estimation rate of the Cole formula (42.9\%) was higher compared to both BSA (31.4\%) and ultrasound (31.4\%), but the correct estimation rates of BSA $(37.2 \%)$ in this subgroup of age were lower than both the Cole formula (54.3\%) and ultrasound $(54.3 \%)$ (Table 4). In patients $\geq 72$ months, the under-, correct- and overestimation rates of the Cole formula, BSA, and ultrasound were all similar. However, the under-estimation rates were higher than in the other age sub-groups for all three parameters. The under-

Table 2. The distribution of correct ETT-OD in subgroups of age

\begin{tabular}{lcccc}
\hline Variable & \multicolumn{5}{c}{ Age group (mo) } \\
\hline Tube size OD $(\mathrm{mm})$ & $\geq 24$ to $<48$ & $\geq 48$ to $<72$ & $\geq 72$ & All patients \\
6.1 & $3(5.3)$ & $1(2.9)$ & - & $4(3.5)$ \\
6.6 & $14(24.6)^{\mathrm{a})}$ & - & - & $14(12.3)$ \\
7.1 & $30(52.6)^{\mathrm{a})}$ & $11(31.3)^{\mathrm{a})}$ & $3(8.6)$ & $44(38.6)$ \\
7.7 & $9(15.8)$ & $15(42.9)^{\mathrm{a})}$ & $\left.5(14.3)^{\mathrm{a}}\right)$ & $29(25.4)$ \\
8.4 & $1(1.7)$ & $8(22.9)$ & $14(40)^{\mathrm{a})}$ & $23(20.2)$ \\
$>8.4^{\mathrm{b})}$ & - & - & $13(37.1)^{\mathrm{a}}$ & - \\
Total & $57(44.8)$ & $35(27.6)$ & $35(27.6)$ & $127(100)$ \\
\hline
\end{tabular}

Values are presented as number (\%).

ETT-OD, the outer diameter of an appropriate uncuffed-endotracheal-tube. a)The number of patients, who required matching ETT-OD size, highlights the most frequent ETT-OD size required in each subgroup of age. ${ }^{\text {b) }} \mathrm{Cuffed}$ ETT of OD: $8.4 \mathrm{~mm}$.

Table 3. The results of univariate linear regression analyses for correct ETT-OD in subgroups of age

\begin{tabular}{lcccr}
\hline Variable & B & $95 \% \mathrm{Cl}$ & $t$ & $P$-value \\
\hline $\begin{array}{l}\text { Age, } \geq 24 \text { to }<48 \mathrm{mo} \\
\text { Weight }\end{array}$ & 0.07 & $0.02-0.13$ & 2.732 & 0.008 \\
$\quad$ Height & 0.22 & $0.04-0.40$ & 2.438 & 0.018 \\
BSA & 0.24 & $0.07-0.42$ & 2.756 & 0.008 \\
Age, $\geq 48$ to $<72$ mo & & & & \\
Weight & 0.07 & $0.02-0.13$ & 1.600 & 0.119 \\
Height & 0.48 & $0.22-0.73$ & 3.782 & $<0.001$ \\
BSA & 0.24 & $0.03-0.46$ & 2.272 & 0.030 \\
Age, $\geq 72$ mo & & & & \\
Weight & 0.07 & $0.02-0.13$ & 3.214 & 0.004 \\
Height & 0.38 & $0.16-0.60$ & 3.563 & 0.002 \\
BSA & 0.28 & $0.12-0.44$ & 3.730 & $<0.001$ \\
\hline
\end{tabular}

ETT-OD, the outer diameter of an appropriate uncuffed-endotracheal-tube; $\mathrm{Cl}$, confidence interval; BSA, body surface area. 
estimation rates of the Cole formula, BSA and ultrasound increased as the age of the children increased (Table 4).

The percentiles of weight- and height-for-age in our patient group were $0.50(0.10-0.75)$ and $0.75(0.25-0.90)$, respectively. The patients below $5 \%$ of weight-for-age and height-for-age were $17.5 \%$ and $8.8 \%$, respectively. The tube-exchange rates did not reveal a significant correlation with weight percentiles $(r=0.019, P=0.842)$ or height percentiles $(r=0.123, P=0.194)$.

Tube size estimations using the Cole formula were lower than the correct ETT-ID by $0.17 \pm 0.41 \mathrm{~mm}$ ( $95 \%$ confidence interval [CI], -0.64 to 0.98 ) (Fig. 2) despite the significant positive corre- lation between the correct ETT-ID and the Cole formula $(r=0.637, P<0.001)$. The 13 patients who were excluded from the size analysis also had unsuccessful predictions obtained from the Cole formula. When those patients were included, in 127 (114+13 patients) patients, tube-exchange rates by using Cole formula were as follows: the correct ETT was found during the first attempt without exchange in $44.9 \%$, during the second attempt in $46.3 \%$, and during the third attempt in $8.8 \%$ of patients. And the percentages in subgroups of age were obtained as follows: the tube size was correctly estimated by Cole formula during the first attempt in 29 patients at age $\geq 24$ to $<48$

Table 4. The frequency distribution of correctly-, under-, and over-estimated tracheal tube size

\begin{tabular}{|c|c|c|c|c|c|c|}
\hline \multirow{2}{*}{ Variable } & \multirow{2}{*}{$\begin{array}{c}\text { Under- } \\
\text { estimated }\end{array}$} & \multirow{2}{*}{$\begin{array}{l}\text { Correctly- } \\
\text { estimated }\end{array}$} & \multirow{2}{*}{$\begin{array}{c}\text { Over- } \\
\text { estimated }\end{array}$} & \multicolumn{3}{|c|}{ Multiple comparisona) } \\
\hline & & & & $P$-value ${ }^{\text {b) }}$ & $P$-value ${ }^{\mathrm{c})}$ & $P$-value \\
\hline \multicolumn{7}{|c|}{ All ages $(n=127)$} \\
\hline Cole formula & $59(46.4)$ & $57(44.9)$ & $11(8.7)$ & NA & NA & NA \\
\hline BSA & $46(36.2)$ & $51(40.2)$ & $30(23.6)$ & $<0.001$ & 0.418 & $<0.001$ \\
\hline Ultrasound & $49(38.6)$ & $54(42.5)$ & $24(18.9)$ & 0.154 & 0.771 & 0.007 \\
\hline \multicolumn{7}{|c|}{ Age $\geq 24$ to $<48 \mathrm{mo}(\mathrm{n}=57)$} \\
\hline Cole formula & $22(38.6)$ & $29(50.9)$ & $6(10.5)$ & NA & NA & NA \\
\hline BSA & $14(24.6)$ & $29(50.8)$ & $14(24.6)$ & 0.021 & $>0.999$ & 0.021 \\
\hline Ultrasound & $15(26.3)$ & $24(42.1)$ & $18(31.6)$ & 0.167 & 0.405 & 0.002 \\
\hline \multicolumn{7}{|c|}{ Age $\geq 48$ to $<72 \mathrm{mo}(\mathrm{n}=35)$} \\
\hline Cole formula & $15(42.9)$ & $19(54.3)$ & $1(2.9)$ & NA & NA & NA \\
\hline BSA & $11(31.4)$ & $13(37.2)$ & $11(31.4)$ & 0.125 & 0.180 & 0.002 \\
\hline Ultrasound & $11(31.4)$ & $19(54.3)$ & $5(14.3)$ & 0.388 & $>0.999$ & 0.125 \\
\hline \multicolumn{7}{|c|}{ Age $\geq 72 \mathrm{mo}(\mathrm{n}=35)$} \\
\hline Cole formula & $22(62.9)$ & $9(25.7)$ & $4(11.4)$ & NA & NA & NA \\
\hline BSA & $21(60.0)$ & $9(25.7)$ & $5(14.3)$ & $>0.999$ & $>0.999$ & $>0.999$ \\
\hline Ultrasound & $23(65.7)$ & $11(31.4)$ & $1(2.9)$ & $>0.999$ & 0.727 & 0.250 \\
\hline
\end{tabular}

Values are presented as number (\%).

NA, not applicable; BSA, body surface area.

a)Cole formula vs. BSA and Cole formula vs. ultrasound, McNemar test. ${ }^{\text {b)}}$ Comparisons of under-estimated rates. ${ }^{\mathrm{c})}$ Comparisons of correctly-estimated rates. ${ }^{d)}$ Comparisons of over-estimated rates.

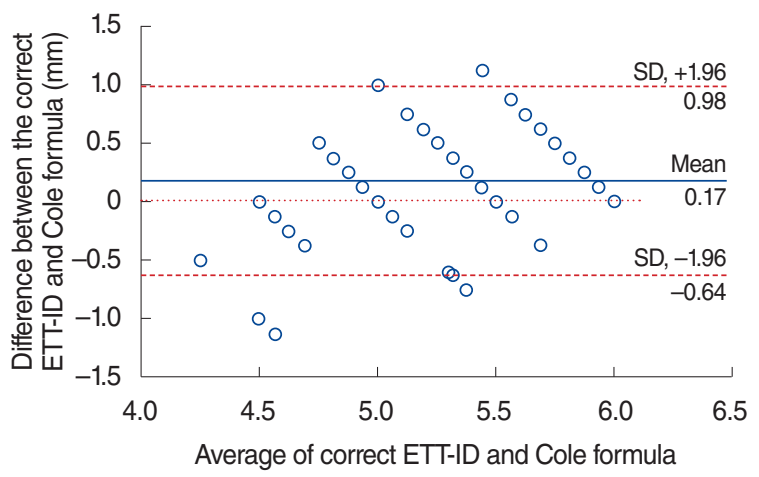

Fig. 2. Bland-Altman diagram showing the magnitude of the difference between the correct ETT-ID and Cole formula ( $n=114$; mean bias, $-0.17 \mathrm{~mm}$ [solid line]; SD, $0.41 \mathrm{~mm}$ ). The dashed lines represent the lower (LL) and upper limits (UL) of agreement: LL, -0.64 mm; UL, 0.98 mm. ETT, endotracheal tube; ID, inner diameter; SD, standard deviation.

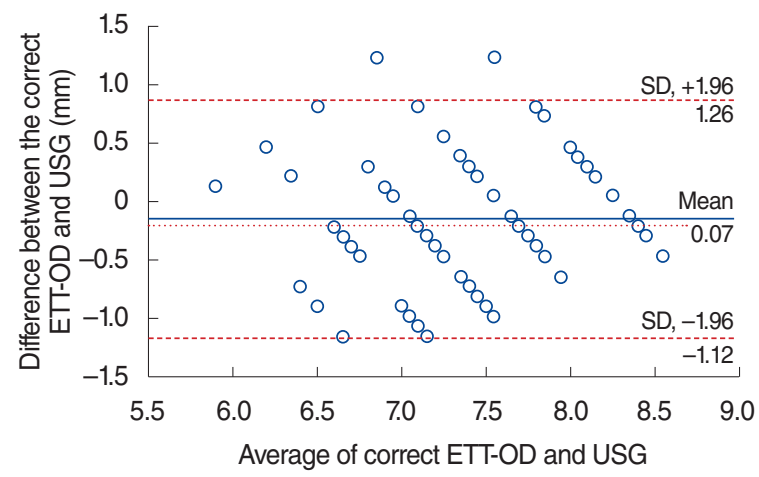

Fig. 3. Bland-Altman diagram showing the magnitude of the difference between the correct ETT-OD and USG ( $n=114$; mean bias, $0.07 \mathrm{~mm}$ [solid line]; SD, $0.61 \mathrm{~mm}$ ). The dashed lines represent the lower (LL) and upper limits (UL) of agreement: LL, $-1.12 \mathrm{~mm}$; UL, $1.26 \mathrm{~mm}$. ETT, endotracheal tube; OD, outer diameter; USG, ultrasonography; SD, standard deviation. 
months ( $n=57,50.9 \%$ ), in 19 patients at age $\geq 48$ to $<72$ months ( $\mathrm{n}=35,54.3 \%$ ) and in nine patients at the age of $\geq 72$ months ( $\mathrm{n}=35,22$ uncuffed and 13 cuffed, $25.7 \%$ ).

The ultrasound measurements were lower than the correct ETT-OD by $0.07 \pm 0.61 \mathrm{~mm}(95 \% \mathrm{CI},-1.12$ to 1.26$)$ (Fig. 3) with a significant positive correlation $(r=0.499, P<0.001)$ even though the correct ETT-OD $(7.35 \pm 0.58 \mathrm{~mm})$ was found to be similar to the ultrasound measurements $(7.42 \pm 0.63 \mathrm{~mm})$ $(P=0.221)$.

\section{DISCUSSION}

Our data suggested that BSA resulted in a similar prediction rate to the Cole formula for patients at age $\geq 24$ to $\leq 96$ months of age. Its correct estimation rate was $40.2 \%$ in all ages, indicating that it is not reliable for clinical use. Age, height, and weight were previously reported to positively correlate with pediatric airway dimensions and correct uncuffed ETT size, similar to our results $[3,8]$. Hofer et al. [9] reported that height-based ETT size estimation using a Broselow tape had a higher predictive value compared to age-based predictions [9]. In a similar study by Daugherty et al. [10], PSS ( $<5 \%$ of height-for-age) children were investigated for the comparison of two methods including age- and height-based formulas for the prediction of ETT size. The height-based estimation of the Broselow tape was comparable to the age-based formula in both PSS and normal children [10]. In our study, height also had a higher correlation with correct ETT size compared to age and weight. In addition, the exchange rates were not different in children $<5 \%$ for both weight-for-age and height-for age.

The correct prediction rate of the age-based Cole formula has been reported to be $47 \%-77 \%$ [6]. In investigations addressing the Cole formula or other age-based estimations, Bae et al. [7] reported 31\%, Schramm et al. [5] reported 24\%, Shibasaki et al. [6] reported $60 \%$, and Daugherty et al. [10] reported $43.2 \%$ for the correct prediction rates of age-based estimations. In those studies, infants and young children $<2$ years of age were also included, which may have constituted a confounding factor. In our study, we included a pediatric group of patients who were $\geq 24$ months ( $\geq 2$ years) of age for whom the exact Cole formula calculation is currently recommended for the prediction of uncuffed ETT size [4]. Our results also showed a similar rate of $44.9 \%$ for the Cole formula to predict the correct uncuffed ETT size. Moreover, the Cole formula had the lowest predictive value in patients $\geq 72$ months of age.

The subglottic transverse diameter measured by ultrasound has previously been shown to have a higher predictive value for ETT size compared to age-based formulas [5-8]. Schramm et al. [5] reported a rate of $48 \%$, Bae et al. [7] reported $60 \%$, and Shibasaki et al. [6] reported $96 \%$ (despite the mean bias of 3.3 $\mathrm{mm}(1.9$ to $4.6 \mathrm{~mm})$. In Schramm et al.'s study [5], ultrasound measurements did not differ significantly from the correct tube size with a mean bias of $-0.02 \mathrm{~mm}$ but were within the limits of agreement (-1.12 to $1.08 \mathrm{~mm}$ ), which was similar to our study. Bae et al. [7] suggested an ultrasound-based estimation as a better alternative to age-based formulas, but it was not reliable for the prediction of appropriate ETT size. In our study, the predictive value of the ultrasound-based estimation was $42.5 \%$, which was similar to previously reported data. Ultrasound may constitute a feasible technique for airway anomalies, but it is an operator-dependent technique, and the predictive value depends on experience despite the suggestion that it is an easy-to-learn technique [6,14]. Subcutaneous fat, probe pressure on the skin, and diagonal slicing may result in errors, but these can be overcome by experience. There are also some drawbacks despite reasonable experience: ultrasound is known to provide distance measurements as accurate as $\pm 1 \mathrm{~mm}$, which may become crucial in the prediction of the size of an ETT in children [8]. Ultrasound was shown to have a higher predictive value, but it was also reported to be useful only for uncuffed tubes [15].

In our study, in patients $\geq 72$ months of age, $40 \%$ required an uncuffed ETT with an OD of $8.4 \mathrm{~mm}$ and $37.1 \%$ needed a cuffed ETT with an OD of $8.4 \mathrm{~mm}$ (Table 2). Therefore, we suggest considering either an uncuffed or cuffed ETT with an OD of $8.4 \mathrm{~mm}$ in patients who are $\geq 72$ months of age. We can also consider using a cuffed ETT with an OD of either 7.7 or 8.4 since it is obvious that the Cole formula more frequently underestimates ETT size in patients over 72 months of age. Similarly, in patients who were $\geq 48$ to $<72$ months of age, $31.3 \%$ required an uncuffed ETT with an OD of $7.1 \mathrm{~mm}$ and $65.8 \%$ $(42.9 \%$ and $22.9 \%)$ needed a larger uncuffed ETT (Table 2). Either an uncuffed or cuffed ETT with an OD of $7.1 \mathrm{~mm}$ may be considered in patients who are $\geq 48$ to $<72$ months of age. In patients who were $\geq 24$ to $<48$ months of age, $52.6 \%$ needed an uncuffed ETT with an OD of $7.1 \mathrm{~mm}, 24.6 \%$ needed a 0.5 $\mathrm{mm}$ smaller ETT, and, $15.8 \%$ needed a $0.5 \mathrm{~mm}$ larger ETT. This patient group may be of clinical importance for future studies that examine BSA specifically to determine the actual correct uncuffed tube. However, it may also be suggested that either an uncuffed ETT with an OD of $6.6 \mathrm{~mm}$ or a cuffed ETT may be considered in this patient group because cuffed ETTs have also been recommended for small children given that cuff pressure monitoring is employed. These age subgroups and their relationship with BSA may lead to future studies.

There are some limitations to our study. Cuffed ETTs have been shown to be used safely in children. However, size estimation studies addressing cuffed tubes may overestimate the success rates of predictive techniques. If a technique predicts a smaller ETT than is actually necessary, the inflation of the cuff with appropriate pressure may overcome the problem concerning the incorrect size estimation. Therefore, in our study, we studied on uncuffed tubes. But, we also suggested using cuffed tubes for subgroups of age and tried to suggest their predicted sizes. How- 
ever, we cannot suggest a definitive predictive technique.

We examined the otherwise healthy patients undergoing elective surgery. We did not do any test to rule out asymptomatic idiopathic or congenital subglottic stenosis. The most common cause of acquired stenosis has been reported to be prolonged intubation, whereas, congenital/idiopathic subglottic stenosis has been reported mostly to be associated with syndromes [16]. Hence, we assessed the history and the presence of syndromes related to any airway pathology and evaluated the presence of any symptoms regarding the airways in order to rule out any airway pathology. Fiberoptic bronchoscopy and imaging techniques are mainly the recommended techniques to rule out stenosis [17]. However, we did not use these techniques to rule out this rare condition. Ultrasound may have been beneficial but the measurement accuracy may differ $\pm 1 \mathrm{~mm}$, which may have misled our measurements. Moreover, in our study, the Cole formula and ultrasound measurements mostly underestimated the size of the tracheal tube, thus we do not think that our results may have been affected by the lack of assessment for stenosis. But, since there are few studies that examine the presence of asymptomatic idiopathic or congenital subglottic stenosis in children, addressing this parameter in future studies will be beneficial.

The high underestimation rate of the Cole formula may have been caused by our preference in favor of smaller size tube when the Cole formula concluded on a value between two sizes. There were nine patients for whom the Cole formula concluded on a size between two. Four of them were at 36 months of age and five of them were at 60 months of age. The tube sizes of three of four patients who were 36 months old and the tube sizes of three of five patients who were 60 months old were underestimated by the Cole formula. We think that the difference of underestimation rates between the age subgroups was not affected.

The correct estimation rates of Cole formula were low for all age groups that the percentages of endotracheal intubations at second and third attempts were to be applied. These second and third attempts may have caused swelling of the mucosa influencing our results. Eight patients at $\geq 24$ to $<48$ months of age, one patient at $\geq 48$ to $<72$ months of age and three patients at $\geq 72$ to $\leq 96$ months of age were overestimated by Cole formula, thus intubated with a smaller tube. We did not exert pressure to advance the ETT in case of resistance and since overestimation was detected to be low, we believe that our results were not affected.

The patients who required a larger ETT rather than an uncuffed ETT with an ID of $6.0 \mathrm{~mm}$ were intubated with a cuffed ETT with an ID of 6.0. Although there is no reason to prefer uncuffed tubes in children $<8$ years of age, this has long been known as the transition age for operator preference of cuffed or uncuffed tubes. Children who required a larger ETT than one with an ID of $6.0 \mathrm{~mm}(\mathrm{OD}, 8.4 \mathrm{~mm})$ were considered old enough to be intubated with a cuffed ETT, and intubation with an uncuffed ETT with an OD of $9.1 \mathrm{~mm}$ (ID, $6.5 \mathrm{~mm}$ ) was not attempted. Instead, these patients were directly intubated with a cuffed ETT with an OD of $8.4 \mathrm{~mm}$. We excluded these patients from the size analysis because we could not be sure of the actual diameter that fit. We believe that our results pertaining to the association between BSA and the uncuffed ETT-OD were not affected by this exclusion.

We addressed the use of BSA for tracheal tube size estimation. The predictive values of either height or age alone are known to be low. We thought that the growth of children, which is evaluated by BSA may also be of use in predicting tracheal tube size. The growth of children is also evaluated by percentiles of weight-for-age and height-for-age. However, we did not find a higher success rate for BSA in predicting ETT size or any correlation between tube exchange rates and growth percentiles. Weight may constitute noise rather than a signal but should be evaluated for its effect on predicting the appropriate size of uncuffed ETT, especially in children with growth retardation defined as $<5 \%$ of weight-for-age.

Ultrasound measurements were performed at the subglottic level immediately below the vocal cords cephalad to the cricoid cartilage. The subglottic area has been defined as the distance between the vocal cords and cricoid cartilage [1,2]. The transverse diameter of this subglottic area was reported to be the narrowest dimension of the airway in children, proving that the contour of the airway in infants and children is different from the previously postulated cone shape [1,2]. The length of this area is difficult to focus on within the ultrasound beam, which may have caused lapse in measurements. In addition, any testretest reliability measurements were not performed. As an operator-dependent technique the ultrasound measurements become more reliable as the experience of the user increases, which may have lowered the successful prediction rates in our study. However, our results were consistent with the results of most previous studies.

We could not present data for postextubation airway complications because they were not an outcome parameter. However, we did not encounter airway complications that required interventions or nebulized steroid therapy. This might have been solved with the $1 \mathrm{mg} / \mathrm{kg}$ of intravenous methylprednisolone administered to our patients who required a tube exchange more than twice. BSA estimated the correct uncuffed ETT-OD successfully at a rate of $40.2 \%$ in patients between age $\geq 24$ and $\leq 96$ months. The BSA, Cole formula, and ultrasonography revealed similar estimation rates. All three parameters had their lowest estimation rates in patients between $\geq 72$ and $\leq 96$ months of age.

\section{CONFLICT OF INTEREST}

No potential conflict of interest relevant to this article was reported. 


\section{ACKNOWLEDGMENTS}

We appreciate the work by Ocal Turgay, Ekinci Saniye, Buyukakkus Berkem, Bulut Yüksel Deniz, Kav Mustafa, Aslan Kav Ezgisu and Ergocen Salih on this study.

\section{REFERENCES}

1. Sirisopana M, Saint-Martin C, Wang NN, Manoukian J, Nguyen LH, Brown KA. Novel measurements of the length of the subglottic airway in infants and young children. Anesth Analg. 2013 Aug;117(2): 462-70.

2.Wani TM, Bissonnette B, Rafiq Malik M, Hayes D Jr, Ramesh AS, Al Sohaibani M, et al. Age-based analysis of pediatric upper airway dimensions using computed tomography imaging. Pediatr Pulmonol. 2016 Mar;51(3):267-71.

3. Dalal PG, Murray D, Messner AH, Feng A, McAllister J, Molter D. Pediatric laryngeal dimensions: an age-based analysis. Anesth Analg. 2009 May;108(5):1475-9.

4. Marciniak B. Growth and development. In: Cote CJ, Lerman J, Anderson BJ, editors. A practice of anesthesia for infants and children. 5th ed. Philadelphia (PA): Elsevier Saunders; 2013. p. 7-21.

5. Schramm C, Knop J, Jensen K, Plaschke K. Role of ultrasound compared to age-related formulas for uncuffed endotracheal intubation in a pediatric population. Paediatr Anaesth. 2012 Aug;22(8):781-6.

6. Shibasaki M, Nakajima Y, Ishii S, Shimizu F, Shime N, Sessler DI. Prediction of pediatric endotracheal tube size by ultrasonography. Anesthesiology. 2010 Oct;113(4):819-24.

7. Bae JY, Byon HJ, Han SS, Kim HS, Kim JT. Usefulness of ultrasound for selecting a correctly sized uncuffed tracheal tube for paediatric patients. Anaesthesia. 2011 Nov;66(11):994-8.

8. Kim EJ, Kim SY, Kim WO, Kim H, Kil HK. Ultrasound measurement of subglottic diameter and an empirical formula for proper endotracheal tube fitting in children. Acta Anaesthesiol Scand. 2013 Oct; 57(9):1124-30.

9. Hofer CK, Ganter M, Tucci M, Klaghofer R, Zollinger A. How reliable is length-based determination of body weight and tracheal tube size in the paediatric age group? The Broselow tape reconsidered. $\mathrm{Br}$ J Anaesth. 2002 Feb;88(2):283-5.

10. Daugherty RJ, Nadkarni V, Brenn BR. Endotracheal tube size estimation for children with pathological short stature. Pediatr Emerg Care. 2006 Nov;22(11):710-7.

11. Neunhoeffer F, Wahl T, Hofbeck M, Renk H, Esslinger M, Hanelt M, et al. A new method for determining the insertion depth of tracheal tubes in children: a pilot study. Br J Anaesth. 2016 Mar;116(3):393-7.

12. Mosteller RD. Simplified calculation of body-surface area. N Engl J Med. 1987 Oct;317(17):1098.

13. Hamill PV, DrizdTA, Johnson CL, Reed RB, Roche AF. NCHS growth curves for children birth-18 years: United States.Vital Health Stat 11. 1977 Nov;(165):i-iv, 1-74.

14. Prasad A, Yu E, Wong DT, Karkhanis R, Gullane P, Chan VW. Comparison of sonography and computed tomography as imaging tools for assessment of airway structures. J Ultrasound Med. $2011 \mathrm{Jul}$; 30(7):965-72.

15. Stafrace S, EngelhardtT,TeohWH, Kristensen MS. Essential ultrasound techniques of the pediatric airway. Paediatr Anaesth. 2016 Feb;26(2): 122-31.

16. Manickavasagam J, Yapa S, Bateman ND, Thevasagayam MS. Congenital familial subglottic stenosis: a case series and review of literature. Int J Pediatr Otorhinolaryngol. 2014 Feb;78(2):359-62.

17. Gnagi SH, Howard BE, Anderson C, Lott DG. Idiopathic subglottic and tracheal stenosis: a survey of the patient experience. Ann Otol Rhinol Laryngol. 2015 Sep;124(9):734-9. 